\title{
Impacto da pandemia covid-19 na formação de residentes de enfermagem, relato de experiência
}

\author{
Impact of the covid-19 pandemic on the training of nursing residents, experience report \\ Impacto de la pandemia covid-19 en la capacitación de residentes de enfermería, informe de experiencia
}

\section{RESUMO}

Objetivo: Relatar a vivência de residentes de enfermagem de um Hospital Federal da Zona Oeste do Rio de Janeiro durante a pandemia. Método: Trata-se de um estudo descritivo de abordagem qualitativa, do tipo relato de experiência, através das atividades no programa de Pós-Graduação em nível de especialização em Clínica Médica e Cirúrgica sob a forma de treinamento em serviço em um Hospital Federal do Rio de Janeiro. Resultados: Os residentes encontram-se diante de um novo cenário com novos desafios, tendo sua atuação profissional frente ao coronavírus. No programa de residência os desafios vivenciados pelos profissionais são capazes de provocar sofrimento ou instituírem forte desenvolvimento psicossocial, porém cada residente desenvolverá sua particularidade em enfrentar os desafios frente a sua atuação. Conclusão: Vivencia-se este cenário, que causou impacto na formação como residente, outro impacto foi a mudança de atividade teórica presencial para educação à distância.

DESCRITORES: Enfermagem; Residência Hospitalar; Pandemia; Infecções por Coronavírus

\section{ABSTRACT}

Objective: To report the experience of nursing residents at a Federal Hospital in the West Zone of Rio de Janeiro during the pandemic. Method: This is a descriptive study with a qualitative approach, of the experience report type, through activities in the Graduate Program at the level of specialization in Clinical Medicine and Surgery in the form of in-service training at a Federal Hospital in Rio of January. Results: Residents are faced with a new scenario with new challenges, having their professional role in the face of coronavirus. In the residency program, the challenges experienced by professionals are capable of causing suffering or establishing a strong psychosocial development, but each resident will develop their particularity in facing the challenges facing their performance. Conclusion: This scenario is experienced, which had an impact on training as a resident, another impact was the change from face-to-face theoretical activity to distance education.

DESCRIPTORS: Nursing; Hospital Residence; Pandemic; Coronavirus Infections.

\section{RESUMEN}

Objetivo: Informar la experiencia de los residentes de enfermería en un Hospital Federal de la Zona Oeste de Río de Janeiro durante la pandemia. Método: Trata-se de um estudo descritivo de abordagem qualitativa, do tipo relato de experiência, através das atividades no programa de Pós-Graduação em nível de especialização em Clínica Médica e Cirúrgica sob a forma de treinamento em serviço em um Hospital Federal do Rio de enero. Resultados: Los vecinos se enfrentan a un nuevo escenario con nuevos retos, teniendo su rol profesional ante el coronavirus. En el programa de residencia, los retos vividos por los profesionales son capaces de provocar sufrimiento o establecer un fuerte desarrollo psicosocial, pero cada residente desarrollará su particularidad al afrontar los retos que afronta su desempeño. Conclusión: Se vive este escenario, que incidió en la formación como residente, otro impacto fue el cambio de la actividad teórica presencial a la educación a distancia.

DESCRIPTORES: Enfermería; Residencia hospitalaria; Pandemia; Infecciones por coronavirus

RECEBIMENTO: 18/09/2020 APROVAÇÃO: 30/09/2020

\section{Eva Natalina Ferreira Costa}

Residente de Enfermagem pela Universidade Federal do Estado do Rio de Janeiro (UNIRIO).

ORCID: 0000-0003-2673-6967

\section{Dayane Martins da Silva Campos}

Residente de Enfermagem pela Universidade Federal do Estado do Rio de Janeiro (UNIRIO) ORCID: 0000-0002-0193-4417 
Flávia Martins Branco

Residente de Enfermagem pela Universidade Federal do Estado do Rio de Janeiro (UNIRIO)

ORCID: 0000-0003-3484-8555

\section{Jessica Brito da Silva Nascimento}

Residente de Enfermagem pela Universidade Federal do Estado do Rio de Janeiro (UNIRIO)

ORCID: 0000-0002-5698-6310

\section{Ana Paula Vital Guerra}

Residente de Enfermagem pela Universidade Federal do Estado do Rio de Janeiro (UNIRIO)

ORCID: 0000-0003-4020-4414

SARS-CoV-2 é um vírus que cau-

sa a doença (coronavírus Disease

2019 ou conhecido também por COVID-19), que gerou uma emergência em saúde pública global, após o surgimento do primeiro caso em dezembro de 2019 na cidade de Wuhan na China. Em 2020 no mês de junho havia mais de 6,4 milhões de casos confirmados e 385.000 mil mortes em todo o mundol.

É uma doença altamente contagiosa e representa riscos relevantes, mas para os profissionais de saúde é amplamente evitável, estes em algumas áreas representam $11 \%$ de todos os casos confirmados de COVID-19. Com um crescente número de mortes atribuídas ao trabalho. $\mathrm{O}$ uso corretamente dos equipamentos individuais pode diminuir consideravelmente o risco de contaminação associada a assistência dos pacientes com COVID-19. Ainda existe pouca evidência que o Equipamento de Proteção Individual (EPI) apresente a melhor proteção. Os treinamentos são fundamentais para paramentação e desparamentarão. Instruções e demonstrações são provavelmente muito vantajosas1.

O abalo psicológico expressivamente elevado pelos profissionais de saúde, que se encontram na linha de frente passando por momentos estressores relacionados aos seus trabalhos, que acarreta em contato social e familiar diminuído, longas jornadas de trabalho, mudanças de protocolos com muita frequência, instruçôes e medidas de segurança e o desenvolvimento de atividades as quais eles podem não estar instruídos. Logo, este momento de aflição vivenciado durante a pandemia COVID-19 pode expressivamente tornar-se em depressão e ansiedade2.
É uma doença

altamente

contagiosa e

representa riscos

relevantes, mas para

os profissionais de

saúde é amplamente

evitável, estes em

algumas áreas

representam 11\%

de todos os casos

confirmados de

COVID-19
No Brasil em meados de janeiro de 2020 o Centro de Operações de Emergência (COE) em saúde pública foi acionado para o novo coronavírus (COVID-19) que se espalhavam pelo mundo. A vigilância epidemiológica de infeção humana pelo SARS CoV 2 será construída e consolidada pela Organização Mundial de Saúde (OMS), com informações trazidas dos países com evidências científicas publicadas3.

A residência em saúde é um programa de pós-graduação em nível de especialização. Denominada por ensino em serviço dividido em 60 horas semanais, com dedicação exclusiva4. $\mathrm{O}$ programa engloba profissionais de diversas categorias que compõe a área de saúde; exemplo enfermagem5.

Os profissionais de saúde necessitam realizar adaptações na assistência para diminuir a contaminação e disseminação do vírus. No entanto como em todas as áreas da vida carecem ser adequadas para um novo modelo de vida. As Unidades de saúde também precisam de ajustes para precaver e inibir o contágio da doença e para contribuir com a recepção e atendimento dos pacientes com suspeita ou confirmação da infecção pelo vírus. Sendo assim, foi de grande valia a tomada de medidas para a reestruturação dos serviços de saúde e conseguinte se manter atualizados com os protocolos fornecidos pelo ministério da saúde6.

Entende-se que dentre todas as mudanças que surgiram e coexistem com a pandemia do COVID-19, os profissionais da área da saúde, como exemplo, os residentes, destacam-se perante tais adaptações e pela atuação como linha de frente. Esses aspectos desenvolveram diversos sentimentos negativos frente a doença, como medo, ansiedade por não saber quando irá acabar, 
a necessidade de se reinventar pela nova situação de saúde, afetando a saúde mental, biológica, física, a situação econômica e os aspectos sociais7.

$\mathrm{O}$ exposto estudo tem como objetivo relatar a vivência de residentes de enfermagem de um Hospital Federal da Zona Oeste do Rio de Janeiro durante a pandemia do novo coronavírus.

\section{METODO}

O presente estudo trata-se de um relato de experiência, do tipo descritivo, com abordagem qualitativa.

Esse tipo de pesquisa segue protocolos que busca proporcionar um conhecimento lógico e cientifico, através de objetivos estabelecidos pelos autores e o uso da metodologia para a obtenção de resultados com altos níveis de evidências, por intermeio da metassíntese, utilizada para associar estudos qualitativos, localizando e dividindo-se em temas, áreas ou tópicos8. A abordagem qualitativa, segue o raciocínio de investigar, avaliar e interpretar os dados9.

$\mathrm{O}$ relato foi descrito durante atividades enquanto enfermeiras residentes e atuantes no segundo ano do programa de Pós- Graduação em nível de especialização em Clínica Médica e Cirúrgica sob a forma de treinamento em serviço em um Hospital Federal do Rio de Janeiro. A seguinte Instituição atende exclusivamente pacientes referentes do Sistema Único de Saúde (SUS).

A Instituição conta com o total de 108 leitos, sendo 10 destinados a emergência, 16 a Unidade de Internação Clínica e 13 leitos Unidade de Terapia Intensiva, estes destinados aos pacientes confirmados ou com suspeita de infecção pelo COVID-19, porém essa instituição não é referência para o tratamento do novo coronavírus.

O relato é baseado na experiência de 5 residentes da área da enfermagem neste atual momento de pandemia, o mesmo foi construído por meio de uma roda de conversa entre os mesmo, abordando principalmente a pergunta sobre como a pandemia os afetou, através das vivências enquanto residentes de saúde e a atuação frente a pandemia do novo coronavírus, também descrevendo sentimentos e dificuldades enfrentadas durante esse período, então a análise da temática será baseado nas falas dos próprios residentes participantes.

Os desafios que os residentes em saúde precisam encarar diariamente, por estar diante de um momento totalmente novo

em suas vidas profissionais, tornase ainda maior diante da situação de saúde e da calamidade a nível mundial, causado por esse novo vírus

Em relação ao tempo, a pesquisa delimita-se a partir das práticas de assistências de residentes de enfermagem durante o seu segundo ano de residência no período de janeiro a julho do ano de 2021.

\section{RESULTADOS}

A motivação desta pesquisa deu-se diante do novo cenário que está sendo vivenciado em Unidades de Treinamento. Através da residência foi possível relacionar a teoria com a prática, fornecendo assim, um conhecimento e aperfeiçoamento profissional de forma única, todavia, a pandemia levou a um cenário de caráter especial, que não espera-se em nenhum aspecto, sendo algo que não é visualizado e/ou ensinado durante a etapa da graduação, ou seja, não é algo visto em teoria, porém foi necessário enfrentar na prática.

Desta forma, os desafios que os residentes em saúde precisam encarar diariamente, por estar diante de um momento totalmente novo em suas vidas profissionais, torna-se ainda maior diante da situação de saúde e da calamidade a nível mundial, causado por esse novo vírus.

Após a administração da primeira dose do imunizante no Brasil, na cidade de São Paulo, contava-se os dias para que chegasse também a vacina na seguinte Unidade de Treinamento. Entretanto, a realidade de vacina para todos, ainda se encontra distante de certa forma, pois inicialmente, apenas 1 residente recebeu a vacina, sendo que a mesma estava lidando diretamente com pacientes diagnosticados ou com suspeita do novo coronavírus.

Apesar de apenas 1 residente ter sido imunizado, surgiu-se no restante da equipe o sentimento de esperança de um futuro com menos riscos do desenvolvimento das formas graves da doença e também esperança pela diminuição do número de internações e mortes pelo coronavírus.

Mesmo diante de diversos empecilhos para imunização de todos os residentes de enfermagem que estavam atuando no hospital e na unidade onde foi realizado o estudo, a situação foi parcialmente resolvida dando ainda mais esperança para que eles trabalhassem com mais segurança.

$\mathrm{O}$ medo entres todos residentes gerou anseios na gravidade de sobrecarga física e emocional, sendo observado que o número de pacientes jovens e sem comorbidades 
aumentava cada vez mais no âmbito hospitalar em que estes profissionais em especialização se encontravam. Neste período a preocupação com os pacientes e si mesmo, o medo, a ansiedade tonaram-se constante na rotina destes residentes em treinamento na unidade.

$\mathrm{O}$ receio, a inquietação e apreensão são sentimentos comuns vivenciados neste momento de pandemia, estamos no segundo ano de combate a este terrível vírus, que altera nossos sentimentos, e não vemos fim para isto.

Ao estar na linha de frente, com alto risco de exposição ao vírus, surge também as preocupações em relação a família e entes queridos, por isso, é necessário ter todo cuidado possível, levamos à risca as orientações sobre paramentação e desparamentação. Destaca-se ainda, a persistência de outras doenças, além do COVID-19, pois as outras patologias não deixaram de existir.

Enfrenta-se as dificuldades de falta de profissionais, a alta demanda nos setores, carga horária exaustiva, extrema cobrança pela calamidade na saúde e falta de equipamento de proteção individual, tivemos uma sensação de insegurança em relação a isso. Luta-se contra um agente invisível que ameaça e mantém como reféns. Ainda temos as perdas irreparáveis, o número alto de óbitos que vem a cada dia, nos deixando de mãos atadas, um cenário de difícil resolução.

Uma questão vivenciada pelo residente é o medo de executar as atividades sem experiência, a pandemia nos trouxe um aumento maior desse sentimento, por diversos motivos, o principal foi a insegurança das informações relacionado a COVID-19.

$\mathrm{O}$ segundo ano de residência nos levou a ter uma segurança maior relacionado as melhores condutas a seguir para tratamento da doença, que pode ser o resultado do avanço dos estudos e até mesmo pelo melhor conhecimento e desenvolvimento que adquirimos da prática em relação ao primeiro ano, apesar disso, sabemos que há um longo percurso para que a saúde alcance um efetivo controle da COVID-19.

Este é um momento de reflexão, pois vivenciamos este cenário nas unidades de Saúde, momento esse que causou impac- to na formação enquanto residentes. Foi necessário a adaptação de uma nova rotina devido ao novo cenário de pandemia. Enfrentamos demandas completamente diferentes e desconhecidas, ações e atendimentos nunca experimentados, pois ainda

Ao estar na linha

de frente, com

alto risco de

exposição ao vírus,

surge também as

preocupaçôes em

relação a família e

entes queridos, por

isso, é necessário

ter todo cuidado

possível, levamos à

risca as orientações

sobre paramentação

e desparamentação

não tínhamos atuado antes frente a uma pandemia.

DISCUSSÃO
Percebe-se um impacto sem precedentes aos profissionais de saúde, provocado pela pandemia do COVID-19, onde uma das condições mentais mais comumente relatadas pelos trabalhadores é a ansiedade. Resultados de estudos mostram que estes profissionais, enfermeiros e médicos, citam um aumento dos níveis de ansiedade, dos quais, até $43 \%$ dos profissionais da linha de frente apresentam um desenvolvimento progressivo dos sinais e sintomas de ansiedade2.

A residência em saúde proporciona uma importante proximidade entre a teoria acadêmica e a prática da área da saúde. A escolha pela residência é devida a essa aproximação da realidade, tornando-se um importante complemento no processo de formação dos profissionais 10 .

Muitas vezes, esses profissionais enfrentam inseguranças no mercado de trabalho, devido os vácuos deixados desde o período de graduação, pela falta da parte prática. $\mathrm{Na}$ residência em saúde, os residentes deixam de ser aluno e passa a ser um profissional atuantel1.

Os Programas de Residência buscam reconhecer a contribuição dos residentes por meio do trabalho multiprofissional e interdisciplinar, visando o desenvolvimento de ações intersetoriais de gestão, atenção, educação e formação em saúde. Nesse período pandêmico o Sistema de Saúde precisou reestruturar suas ações para enfrentamento da doença e o residente como parte integrante do processo de trabalho precisou de readequar, vivenciando desafios capazes de provocar sofrimento ou instituírem forte prazer e desenvolvimento psicossocial 10.

Os profissionais encontram-se diante de um novo cenário com novos desafios, tendo sua atuação profissional frente ao novo coronavírus. No programa de residência os desafios vivenciados pelos profissionais são capazes de provocar sofrimento ou instituírem fonte de prazer e desenvolvimento psicossocial. Porém cada residente desenvolverá sua particularidade em enfrentar os desafios frente a sua atuação na Unidade de Treinamento9.

As mudanças extremas vivenciadas pelos profissionais de saúde neste período de pan- 
dêmico provocou situações psicológicas expressivas. Além do medo de morrer, trouxe também implicações para outras esferas como o fechamento de escolas, empresas, organização familiar, órgãos públicos, mudança na rotina, isolamento e distanciamento social12-13.

No entanto o momento econômico tem causado grande repercussão devido a insegurança motivada pelo sentimento de abandono, desamparo e a incerteza de trabalho14.

Após um ano de espera de algum tipo de solução, no dia 17 de janeiro do ano de 2021, a primeira brasileira foi imunizada com a vacina para o Covid-19 foi uma enfermeira que atuava na Unidade de Terapia
Intensiva do Instituto de Infectologia Emílio Ribas, em São Paulo. Após a liberação da Agência Nacional de Vigilância Sanitária (ANVISA), com restrições, iniciou-se o uso emergencial da vacina do Butantan, produzida com um laboratório chinês Sinovac15.

\section{CONCLUSÃO}

A experiência advinda da vivência diária da rotina nos serviços de saúde possibilita aos residentes amadurecimento profissional, à amplificação de competências diminui as probabilidades de equívocos, além de segurança e autonomia para melhor tomada de decisões frente a situações complexas no dia-a-dia.
Porém, mesmo sendo uma experiência enriquecedora, o combate à pandemia tem ensinado que mesmo na adversidade, as equipes de saúde podem se reformular e reorganizar as ações planejadas para garantir que a atenção à saúde seja prestada aos usuários da melhor maneira possível, mas que é necessário atender para as dificuldades, sensações e sentimentos dos profissionais da saúde, pois os mesmos referem sofrimentos.

Nessa perspectiva, espera-se que a experiência descrita permita ao leitor a reflexão sobre a importância da atuação profissional dos residentes nos ambientes hospitalares e que possa provocar o entendimento sobre os sentimentos e dificuldades enfrentadas pelos profissionais.

\section{REFERÊNCIAS}

1 Tabah $A$, et al. Personal protective equipment and intensive care unit healthcare worker safety in the COVID-19 era (PPE-SAFE): An international survey. J Crit Care, vol. 59, pág. 70-75, oct., 2020.

2 Javier $S$, et al. Prevalence of anxiety in health care professionals during the COVID-19 pandemic: A rapid systematic review (on published articles in Medline) with meta-analysis. Progress in Neuro-Psychopharmacology and Biological Psychiatry, vol. 107, 2021.

3 Brasil. Ministério da Saúde. Protocolo de manejo clínico da Covid-19 na Atenção Especializada. Departamento de Atenção Hospitalar Domiciliar e de Urgência, $1^{\circ}$ edição, 2020.

4 Brasil. Ministério da Saúde. Ministério da Educação. Portaria interministerial $n^{\circ}$ 45, de 12 de janeiro de 2007. Dispõe sobre a Residência Multiprofissional em Saúde e a Residência em Área Profissional da Saúde e Institui a Comissão Nacional de Residência Multiprofissional em Saúde. Diário Oficial da União, Brasília, 12 jan., 2007.

5 Brasil. Ministério da Saúde. Resolução n² 287 de 08 de outubro de 1998. Diário Oficial da União, Brasília, 08 out., 1998.

6 Lima DS, et al. Recomendações para cirurgia de emergência durante a pandemia do COVID-19. J. Health Biol Sci., Fortaleza, vol. 08, n. 01, pág. 1-3, 2020.

7 Caldas GRF, et al. Novo Coronavírus: Impacto na saúde mental dos profissionais de saúde. Research, Society and Development, v. 10, n. 5, 2021.

8 de Oliveira G, Moreira AP, Floriano LSM, Bordin D, Bobato GR, Cabral LPA. Impacto da pandemia da Covid-19 na formação de Residentes em Saúde. Brazilian Journal Of Development, Curitiba, vol. 06, n. 11, pág. 90068-90083, nov., 2020.
9 Fernandes MNS, et al. Sofrimento e prazer no processo de formação de residentes multiprofissionais em saúde. Rev. Gaúcha Enferm., Porto Alegre, vol. 36, n. 04, pág. 90-97, 2015.

10 Fernandes MNS. Prazer e Sofrimento no processo de formação de residentes multiprofissionais em saúde. 118 f. Tese (Doutorado) - Curso de Enfermagem, Universidade Federal de Santa Maria, Santa Maria, 2013.

11 Alvarenga GAB, Galvão EFC, Takanashi SLY. Percepção dos residentes do processo avaliativo e seus instrumentos na residência multiprofissional na atenção integral em ortopedia e traumatologia. Revista Exitus, vol. 09, n. 01, pág. 455-479, jan., 2019.

12 Ornell F, Schuch JB, Sordi AO Kessler FHP. "Medo pandêmico" e COVID-19: Ônus e estratégias para a Saúde Mental. Braz J Psychiatry, vol. 42, n. 03, pág. 232-235, 2020.

13 Lima RC. Distanciamento e isolamento sociais pela Covid-19 no Brasil: impactos na saúde mental. Physis: Revista de Saúde Coletiva, vol. 30, n. 02, 2020.

14 Noal D da S, Passos MFD, Freitas CM. Recomendações e orientações em Saúde Mental e Atenção Psicossocial na Covid-19. Fundação Oswaldo Cruz (FIOCRUZ), 2020. Disponível em: https:// www.fiocruzbrasilia.fiocruz.br/wp-content/uploads/2020/10/livro_ saude_mental_covid19_Fiocruz.pdf

15 Enfermeira que recebeu a primeira dose do imunizante no Brasil. Jornal UOL, 2020. Disponível em: https://noticias.uol.com.br/saude/ ultimasnoticias/redacao/2021/01/17/enfermeiradespnegramoradoradeitaquerae1 vacinadanobrasil.htm?cmpid=copiaecola. Acesso 28/04/2021. 